\title{
Advances in petroleum industry seismic acquisition technology
}

Dave Monk, Apache

When it comes to acquisition of seismic data onshore there are really only 2 basic attributes which can be addressed which impact how data is acquired.

The first is the acquisition geometry, the relationship between sources and receivers. This of course includes the issues of arrays at both the source and receiver end of the raypath. Recent developments in acquisition technology have led to far more flexability in receiver geometries, and the indication is that we will have the capability of recording up to 1 million traces in each shot record within 5 years. Additionally we have seen development of simultaneous source methods which have allowed far greater flexability in source geometry as well.

The second fundamental issue goiverned by acquisition technology is the bandwidth of the data acquired. This is limited by both source and receiver, and recent developments have aimed at extending the bandwidth of both as we move towards more sophisticated processing including full waveform inversion. Recently there has been considerable work in extending the low frequency components of the bandwidth.

In this presentation a road map of how acquisition may evolve is presented which highlights both the geometry and bandwidth changes we are likely to see in the future through new technology. 